\title{
NICE prostate cancer clinical guideline: implications for primary care
}

Prostate cancer is the commonest cancer in men, with 32000 new cases annually. Its incidence is increasing, largely as a result of an increase in prostate specific antigen (PSA) testing over the last two decades. ${ }^{1}$ However prostate cancer deaths are relatively stable at 10000 deaths a year, suggesting that the increase in PSA tests has resulted in more diagnoses of early-stage disease whose treatment does not have a significant impact on mortality. It is a disease of older age, being rare in under 50 s, with a peak prevalence in the seventh decade. ${ }^{1}$ Prostate cancer has a variable natural history, with many cancers remaining relatively indolent and causing few, if any, clinical problems, and others running a more aggressive course. This has led to difficulties and uncertainties in evaluating the effectiveness and cost-effectiveness of both treatments, and monitoring protocols, for various forms of the disease.

One consequence of the increasing prevalence is that there are now many more men living with the disease and needing some form of ongoing monitoring. The purpose of this monitoring is to: detect recurrence or spread of disease (through reporting of symptoms, clinical examination, PSA testing, and other investigations); to assess the effects and side-effects of treatments; to detect and manage psychosocial problems; and to manage comorbidities. Over recent years there have been trends for men to be 'discharged' to primary care for monitoring if secondary care consider that they only needed an annual PSA. Issues regarding treatment and ongoing management of prostate cancer in the UK are the subject of a new clinical guideline. $^{2}$

The guideline only covers the management of prostate cancer. This means that many other areas are not covered, such as issues of service organisation and guidance on the symptomatic management of urinary symptoms, sexual function, bowel problems, and the management of comorbidity. Other guidance covers primary care diagnostic issues and supportive and palliative care..$^{3,4}$ Much of the guideline concerns procedures in secondary care, and the value of this to GPs will be in helping and advising patients about treatment options. However, much of the detail is only available in the full guidance (112 pages) and not in the quick reference guide (12 pages). Even in the full version some facts that GPs may want to have to hand are missing; for example the $5 \%$ absolute risk reduction with radical prostatectomy compared with watchful waiting is useful, but the associated increased risk of erectile dysfunction and incontinence is not quantified. The most important and relevant issues for primary care are in providing (some) clarity relating to recommendations for different management strategies, including the place of, and process of, watchful waiting, active surveillance, and followup of men having had local treatments 2 years post-treatment with a stable PSA. However, many of the recommendations are inevitably based upon consensus opinion of the guideline development group rather than robust evidence. While this is not a huge weakness (there are many precedents for this in other clinical areas), it does introduce an element of doubt, and perhaps choice, in GPs' minds.

So, what are the key implications for primary care? Follow-up is the area of most consequence. It is important to distinguish between 'watchful waiting' and 'active surveillance'. Most GPs will not think of these as separate, and this has not been helped by secondary care using the terms with imprecision, and by the practical management issues of the two strategies being relatively similar. 'Watchful waiting' is recommended for local disease when there are no clear benefits of radical treatments. This is because the potential harms of surgery or radical radiotherapy outweigh any potential benefits, and it is usually therefore a treatment offered to older men and those with significant comorbidity, who are far more likely to die of something else or not be physically strong enough for radical treatments. Negative connotations associated with the term watchful waiting are not always helpful because it is sometimes equated to 'no treatment' ${ }^{5}$ and this can be a difficult message to convey to patients and their families. 'Active surveillance' is recommended for the management of men with low-risk localised disease who would potentially stand to benefit from radical treatments in the face of disease progression, and is therefore different from watchful waiting. Active monitoring of disease through a formal regular process of biochemical tests and biopsies is intended to identify disease progression and referral back to the multidisciplinary team (MDT).

The guideline recommends that in watchful waiting men should be followedup in primary care, and that this should be to monitor symptoms of progressive disease (predominantly urine outflow obstruction) and to perform an annual PSA test. Men whose disease does develop symptomatically or biochemically, should then be referred back to the MDT for consideration of palliative radiotherapy or hormonal treatments. These men will only be seen for their prostate cancer in primary care; hence all supportive and psychosocial care will need to be provided in this setting. For active surveillance men are recommended to receive secondary care biopsies at intervals, with PSAs at intervals in between; the frequency and location of testing is not specified. For men who have received local treatments and are 2 years post-treatment with a stable PSA, the guideline recommends 
that follow-up should be outside of hospital, with at least an annual PSA.

What is explicitly missing from the guideline is any notion of exactly what primary care should actually do (whether watchful waiting, active surveillance, or stable post-treatment) and how they should do it. For example, how 'patientinitiated' should 'annual' follow-up in watchful waiting be, and how vigorous should primary care be in seeking symptoms of urinary obstruction? And how should annual PSAs for active surveillance be organised between primary and secondary care and specialist nurses? There is the potential for confusion, duplicate testing and for some men to be lost to follow-up. ${ }^{6}$ Primary care needs to be aware that the evidence for the benefit of annual PSAs in watchful waiting is thin, and somewhat counterintuitive to the notion of watchful waiting.

While NICE guidance may be regarded by some as 'cookbook medicine' that reduces patient-centred approaches, the flexibility afforded to primary care in this guidance is welcomed. While the evidence base for many of its recommendations is small, the scope of the guideline should be applauded. In taking this guideline forward, primary care organisations (with input from local GPs) need to agree local policies and protocols with cancer networks and then disseminate to primary care health professionals. There will be no 'one size fits all' and local, workable policies are essential. Information exchange between care sectors must be of the highest quality. Picking up the pieces following a secondary care consultation where, for example, watchful waiting has been the recommended outcome, and where both the patient and the GP are unclear why there is a perception that 'no treatment' is being offered is no easy task. Structured and prompt communication explaining the treatment option, justification for this, information given to patient and carers, and action required by primary care is essential. While the result of one major trial is awaited, ${ }^{7}$ there is a clear need to undertake more rigorous evaluations of different models of management of prostate cancer in primary care.

\section{Richard D Neal,}

Senior Lecturer in General Practice, Cardiff University, Wales.

\section{REFERENCES}

1. Cancer Research UK. CancerStats Key Facts on
Prostate Cancer.

http://info.cancerresearchuk.org/cancerstats/types/p rostate/ (accessed 4 Aug 2008).

2. National Institute for Health and Clinical Excellence. Prostate cancer diagnosis and treatment. Cardiff: National Collaborating Centre for Cancer, 2008.

3. National Institute for Clinical Excellence. Supportive and palliative care. London: NICE, 2004

4. National Institute for Clinical Excellence. Referral guidelines for suspected cancer. London: NICE, 2005

5. Donovan J, Mills N, Smith M, et al. Improving design and conduct of randomised trials by embedding them in qualitative research: ProtecT (prostate testing for cancer and treatment) study. BMJ 2002; 325: 766-770.

6. Neal RD. Management of prostate cancer in general practice. Trends in urology, gynaecology and sexual health 2008: in press.

7. University of Bristol. ProtecT Study (Prostate testing for cancer and Treatment). http://www. epi.bris.ac.uk/protect/ (accessed 4 Aug 2008).

DOI: 10.3399/bjgp08X330708

\section{ADDRESS FOR CORRESPONDENCE}

Richard D Neal,

Senior Lecturer in General Practice, North Wales Clinical School, School of Medicine Cardiff University, Gwenfro Unit 5,

Wrexham Technology Park,

Wrexham LL13 7YP.

E-mail: nealrd@cf.ac.uk 